\title{
Self-Control Cyclic Access with Time Division - A MAC Proposal for The HFC System
}

\author{
S.M. Jiang, Danny H.K. Tsang, Samuel T. Chanson \\ Hong Kong University of Science \& Technology \\ Clear Water Bay, Kowloon, Hong Kong
}

\begin{abstract}
The IEEE 802.14 standard committee is currently working on a project to find a cost-effective means of providing access to integrated networks for people to enjoy multimedia programs and to work at home. An advanced system based on the CATV system called Hybrid Fiber Coax is being studied. Since some properties of the HFC system preclude the possibility of directly using existing medium access control protocols for its data link layer, a MAC scheme based on time division is discussed in this paper.
\end{abstract}

\section{Introduction}

As ATM technology progresses, researchers are looking for a cost-effective means of providing home access to integrated networks so that people can enjoy multimedia programs and work at home. Due to the large geographical coverage of existing CATV systems, an advanced system based on CATV called Hybrid Fiber Coax (HFC) is being studied by the IEEE 802.14 Project Committee [1]. This system will likely use ATM technology to provide multiple services to home such as telephone, TV and information retrieval. In the HFC system, the downstream band and upstream band operate asymmetrically at different frequencies, and a node has only one link to each bus. Therefore, the HFC system may be abstracted into a logical dual-branch-bus based network consisting of a distributing hub (called headend in this paper) and the fiber nodes (or simply nodes) as shown in Figure 3. In the downstream direction (from the headend to the nodes) broadcast mode is used, and the headend is the only transmitter. This makes control of the downstream band simple. In the upstream direction (from the nodes to the headend), the nodes share the upstream band for transmission, and contention access mode is used. To allow each node to have equal opportunity to share the upstream band, an efficient medium access control (MAC) protocol is necessary. This protocol has to be cost-effective and efficient for long distance communication, supporting a wide range of transmission rates as well as both analog and digital broadband services [2]. Unfortunately, these properties and requirements make it difficult to use existing MAC protocols directly for the following reasons:

1) The ring-based protocols such as Token-Ring [5], FDDI [5], ATMR [3] and Orwell [4] are not suitable for the dualbranch-bus topology required by the HFC system. On the other hand, both CSMA/CD and Token-Bus [5] are suitable for the HFC topology. However, CSMA/CD cannot guarantee bounded delay and is only suitable for relatively short distances. The weakness of Token-Bus is that it requires the users to remain on-line to be included in the logical ring which needs to be reconfigured whenever users are added or removed from the network.

2) It is difficult to use MAC protocols based on the slotted media approach like DQDB [5] and CRMA [6]. These protocols often require a node to have two links to each bus for data insertion. Moreover, DQDB needs two headends and CRMA requires a folded bus while there is only one logical headend and the topology is required to be dual-branch-bus in the HFC system. Although the two logical buses in the HFC system can be looped to form a folded bus like CRMA, the outbound bus (from the headend to the nodes) is reserved for transmission, and the inbound bus (from the nodes to the headend) for receiving in the folded-bus CRMA. This is opposite to the HFC system where the downstream band is for the users to receive data and upstream band for the users to transmit data.

With regards to the special position of the headend in the HFC system, a MAC scheme based on centralized control is a natural choice. One such scheme is the traditional polling system such as Roll-call Polling [8]. However, these systems have been designed for low or medium speed data applications under the assumption that the propagation delay is negligible compared to the transmission time. This assumption is not true in high speed networks [7]. In this paper, we will discuss a new MAC scheme called Self-Control Cycle Access with Time Division (SCCA/TD).

The rest of the paper is organized as follows. The principle of SCCA/TD is introduced in Section 2, and simulation studies on this prototype are give in Section 3. Finally Section 4 concludes the paper.

\section{Introduction to SCCA/TD}

SCCA/TD is a reservation based MAC scheme which allows the nodes to share the upstream bandwidth. A node normally needs to make requests for bandwidth through the upstream band. The headend will respond to the requesting nodes through the downstream band. The upstream bandwidth is divided into equal time segments called 'cycles'. A cycle is divided again into fractions which are assigned to the nodes according to their requests. As shown in Figure 1, the same epoch of the cycle is repeated periodically as long as the requests of the nodes are unchanged. That is, the quota and position of the bandwidth allocation to a given node in each cycle are not changed. In this way, a node can access the medium simply knowing the relative position of its assignment in the cycle. The granted bandwidth can be used by the nodes for transmitting data or submitting requests.

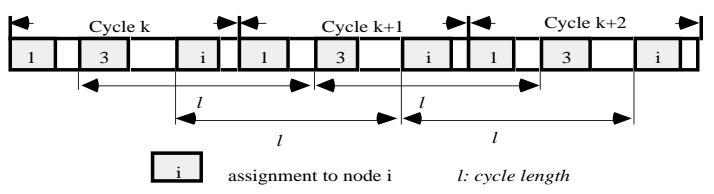

Figure 1 The principle of SCCA/TD

\subsection{Cycle Structure}


A cycle is divided into the static reservation segment (SRS) and the dynamic reservation segment (DRS) as shown in Figure 2. SRS gives each node a basic bandwidth assignment periodically in each cycle. A node can use the SRS assignment to submit requests or transmit small amounts of data. DRS, on the other hand, provides bandwidth dynamically to the nodes according to their requests. The advantage of using SRS is that it provides the nodes quick access to the medium. However, if a node has nothing to transmit, its assignment will be wasted. Requiring several nodes to share the same assignment alternately can improve efficiency. When a node has a large amount of data to transmit, it needs to get the requested bandwidth from DRS. To this end, the node submits a request to the headend carried by a special packet containing the node's identifier, transmission speed, request type and other related parameters. The headend will respond with a special confirmation packet.

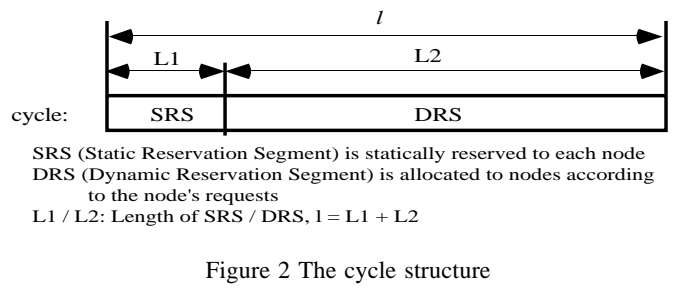

There are two types of requests that a node can submit to the headend: Gross Bandwidth Request (GBR) and Cyclic Bandwidth Request (CBR). With GBR, a node informs the headend of the total amount of bandwidth it needs, and the headend serves the request in consecutive cycles if the request cannot be satisfied within a single cycle. In this case, the requesting node will get the total required bandwidth in multiple cycles, each providing only a fraction of the total required bandwidth. With CBR, a node informs the headend of the bandwidth it requires in each cycle. If the request is granted, the node gets the same quota of bandwidth in the same relative location in each subsequent cycle until the end of transmission. When the node finishes transmission, it has to send a message to the headend to relinquish its DRS reservation. The first type of request is suitable for bursty traffic such as data while the second type is suitable for periodic traffic such as voice and video.

For both SRS and DRS, the nodes can adopt the selfcontrol cyclic access mechanism to use the bandwidth after receiving confirmation without additional control from the headend. That is, the headend notifies the requesting node the starting time of its bandwidth assignment, the quota of bandwidth that the node can use in each cycle and the cycle length, i.e., the period in which the node can use its assignment until either the expiration of its grant (for GBR request) or the end of transmission (for CBR request). This scheme can be implemented with timers or counters for fixed length MAC protocol data units.

\subsection{Scheduling Schemes}

Collisions burden networks with extra work for collision detection and resolution, and wastes bandwidth. This is especially undesirable in high-speed networks. Therefore, SCCA/TD is designed to be collision-free. The scheduling done by the headend is the key to collision avoidance. The bandwidth allocation mechanism has to maintain fairness for the nodes to share bandwidth while avoiding collision to improve network utilization. There are many alternatives for the allocation policy. A simple scheme would be for the headend to collect the requests and serve them one by one in round robin. This scheme is used in the simulation studies below. In the following, we discuss two collision avoidance approaches. The following parameters are used in the discussion:

$n$ - total number of nodes in the network (see Figure 3);

$m$ - total number of nodes simultaneously sharing the same cycle;

$l$ - cycle length;

$d(\mathrm{i}, \mathrm{j})$ - distance between nodes i and $\mathrm{j}$;

$d$ - distance between nodes 1 and $\mathrm{n}$, i.e., maximum propagation delay;

$\mu$ - bandwidth utilization measured in terms of the total time used for transmission within a cycle over the cycle length.

(for simplicity, $l, d(\mathrm{i}, \mathrm{j})$ and $d$ are all measured in time units)

a) The Efficient Approach (EA)

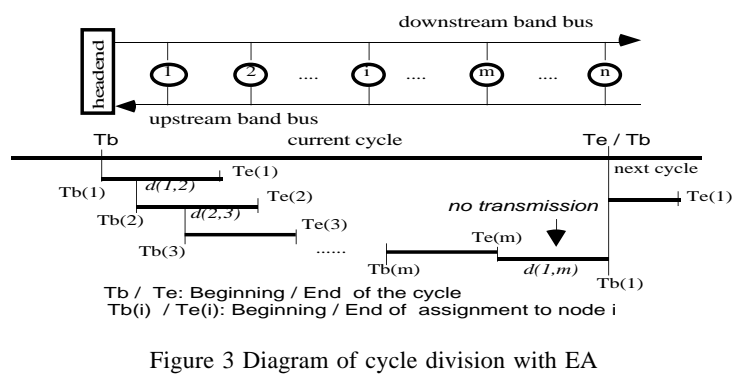

For this approach, we suppose that the signal propagation in the upstream band bus is unidirectional towards the headend, and the sequence of bandwidth assignment to the nodes in each cycle is in accordance with the sequence of their physical positions in the network. In this case, the cycle always begins with the assignment to the node closest to the headend. The propagation delay between adjacent nodes is taken into account to maximize bandwidth utilization which can go up to 1 . This can be explained by Figure 3, where the cycle is fully shared by $m$ active nodes.

Notice that an active node $i+1$ can advance its transmission by $d(\mathrm{i}, \mathrm{i}+1)$ ahead of the end of transmission of node $\mathrm{i}$ due to propagation delay. The total time advanced within a cycle is equal to $\sum d(\mathrm{i}, \mathrm{i}+1)=d(1, \mathrm{~m}) \quad(\mathrm{i}=1,2, . . m$ $1)$. When the last active node $m$ finishes transmission, time $d(1, \mathrm{~m})$ is required to allow its traffic pass through all the downstream nodes before the beginning of the next cycle. This time is compensated by the total time advanced by all the nodes. Therefore, EA can give high bandwidth utilization independent of network size and cycle length. However, if no bandwidth in a suitable position in the cycle is available to a new requesting node, the headend has to shift the positions of some existing assignments in order to accept new requests. For example, as shown in Figure 4, if nodes 3, 4 and 8 become active, there is no free segment suitable to them even though there are several free segments in the cycle. By shifting the bandwidth assignments for nodes 1,2 and 7 to the left (occupying some of the unassigned space), some spaces are made available for the new requesting nodes in the right position. In this case, the headend needs to send messages to the affected nodes informing them their new 
bandwidth assignments in the cycle. Shifting bandwidth assignments can provide more flexible and efficient bandwidth allocation at the expense of complexity.

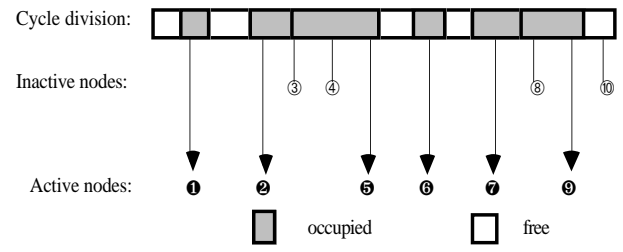

Figure 4 Assignment in a cycle versus the physical position of the nodes

A simple implementation of EA called quasi-EA (qEA) is that when allocating an assignment in the cycle for a node, the position of the assignment in the cycle is determined by the node's physical position in the network to avoid irregular cycle divisions.

\section{b) The Simple Approach (SA)}

With this approach, the relative physical positions of the nodes in the network are not taken into account. The maximum distance (i.e., $d$ ) is used as the distance between any pair of nodes. In this case, when an active node finishes its assignment in a cycle, the next active node has to wait $d$ time units before starting its transmission. $\mu=1-m * \Delta$, where $\Delta=d / l$, is the bandwidth wastage per active node due to the gap placed between adjacent assignments for avoiding collision. As shown in Figure 6, bandwidth utilization is inversely proportional to the number of active nodes for a given cycle length and network size. The maximum bandwidth utilization is bounded by $1-\Delta$. Therefore, this approach is suitable for small size networks only because it is easy to get a small $\Delta$ with long cycles in this case.

\section{Simulation Studies}

As mentioned in Section 2.1, there are two types of requests that a node can make, i.e., GBR and CBR. With $\mathrm{CBR}$, a node can get the guaranteed bandwidth in every cycle if its CBR request is confirmed. This is similar to GBR when a large GBR request is divided into multiple fragments served by several consecutive cycles. Therefore, our simulation is concerned with GBR requests only.

\subsection{Simulation Model}

The simulation model consists of 25 nodes with equal distance between any two neighboring nodes (s). The network size (NS) is equal to $24 * \mathbf{s}$ (assuming the distance between node 1 and the headend is 0 ). The traffic model of each node is a bursty pulse train [9] as shown in Figure 5. The delay between two consecutive bursts and the number of cells in a burst follow respectively the exponential and geometric distributions. The network capacity is set to $155.52 \mathrm{Mbps}$ corresponding to OC-3, which results in the cell length (CL: time to transmit an ATM cell) of $2.726 \mu \mathrm{s}$. The static reservation for each node is set to $\mathbf{s}$. The length of the static reservation segment in a cycle, $\mathrm{L} 1$, is equal to $25 * \mathbf{s}$. The mean cell arrival rate from a node (MCR) can be computed by $\mathrm{BL} /(\mathrm{DB}+\mathrm{BL} * \mathrm{IC})$. The cell transmission rate given by the network is equal to $1 / \mathrm{CL}$, which is shared by 25 nodes so that each node gets $1 /(25 * \mathrm{CL})$. The offered load (OL) is defined as MCR / [1 / $(25 * \mathrm{CL})]=25 * \mathrm{CL} / \mathrm{MCR}$. Given $\mathrm{CL}, \mathrm{OL}$ and $\mathrm{BL}, \mathrm{DB}=(25 / \mathrm{OL}-1) * \mathrm{BL} * \mathrm{CL}$.

The main performance index to be investigated is bandwidth utilization $\mu$. The request response time is not measured since a node can continue to make requests even after the previous ones have not been honored; and the headend may honor several requests for a given node at the same time. Table 1 lists the main parameter values used by the different simulations (NS = network size, BS = buffer size, $l=$ cycle length, $\mathrm{OL}=$ offered load, $\mathrm{BL}=$ burst length, $\mathrm{PV}=$ parameter to be varied). The default $\mathrm{M}$ setting is (L2 I) for the SA scheme with $\mathrm{I}=d$, and L2 / 25 for the qEA scheme.

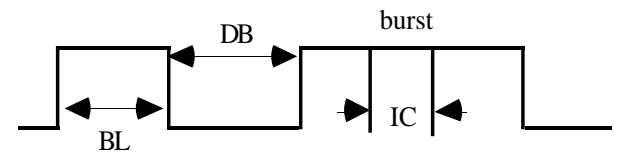

DB: mean delay between two burrsts (exponential distribution)

BL: mean number of cells in a burst (geometric distribution with probability $=1.0 /[\mathrm{BL}+1])$

IC: interval between two cells (constant equal to a cell length)

Figure 5 Burst pulse train traffic model

\begin{tabular}{|l||l|l|l|l|l|}
\hline Figure & $\begin{array}{l}\text { NS } \\
(\mathbf{k m})\end{array}$ & $\begin{array}{l}\text { BS } \\
\text { (cell) }\end{array}$ & $\begin{array}{l}\text { (cell) } \\
\text { (c) }\end{array}$ & $\begin{array}{l}\text { BL } \\
\text { (cell) }\end{array}$ \\
\hline \hline Fig.6 & 13 & 2500 & PV & 1.0 & 30 \\
\hline Fig.7 & 13 & 2500 & PV & PV & 30 \\
\hline Fig.8 & 13 & PV & 300 & 0.75 & PV \\
\hline Fig.9 & 13 & PV & 300 & 0.75 & PV \\
\hline Fig.10 & 13 & PV & 300 & 0.75 & PV \\
\hline Fig.11 & PV & 2500 & PV & 1.0 & 30 \\
\hline Fig.12 & PV & 2500 & PV & 1.0 & PV \\
\hline
\end{tabular}

Table 1 Parameter setting for the different simulations

\subsection{Simulation Results}

This subsection is organized as follows: Figures 6 to 10 investigate the effects of some parameter settings (e.g., request threshold, offered load and buffer size) to the performance of SA and qEA schemes; and Figure 11 compares the performance between SA and qEA. Although qEA shows better performance than SA in many cases as shown below, the main advantages of SA are its simplicity and applicability to wireless networks. The simulation results are obtained using the simulation package BONes DESIGNER V 2.6 from Alta Group ${ }^{\mathrm{TM}}$. Each point on the graphs took about 3 hours to simulate on a SPARC-5 machine.

Figure 6 shows the effect of request threshold (T) to bandwidth utilization $(\mu)$ for SA and qEA. It is observed that $\mathrm{T}$ has very little effect on $\mu$. For SA, a reasonable cycle setting (e.g., $\mathrm{CL}=300$ ) can produce a good $\mu$. For qEA, the cycle length is 500 cells and $\mathrm{M}$ is set to $15,19,25$ (average setting) and 451 (maximum setting) cells respectively. Note that SA can almost achieve the same $\mu$ as qEA, but the number of simultaneous active nodes in SA is limited to 1 or 2 by the maximum $M$ setting (i.e., $M=L 2-I$ ) while that in qEA is equal to $\mathrm{N}$ (e.g., $\mathrm{N}=25$ in this simulation model).

Figure 7 depicts bandwidth utilization $(\mu)$ versus offered load (OL) for different cycle lengths $(l) . \mu$ at $\mathrm{T}$ is the theoretical maximum value $(\mathrm{TM} \mu)$ for a given network size and cycle length according to the analyses in Section 2.2. Note that it is easier for traffic to fill a shorter cycle (e.g., $l=$ 100) rather than a longer one. However, increasing $\mu$ with 
increasing OL in SA with a short cycle is limited by the $\mathrm{TM} \mu$ which is smaller than 1 in SA. It is possible to have a high $\mu$ in qEA since the TM $\mu$ is equal to 1 . In addition, different cycles in qEA lead to the same $\mu$ in the case of heavy load since $\mu$ given by qEA is not influenced by the cycle setting but mainly depends on the traffic pattern of the nodes like the EA scheme mentioned in Section 2.2.

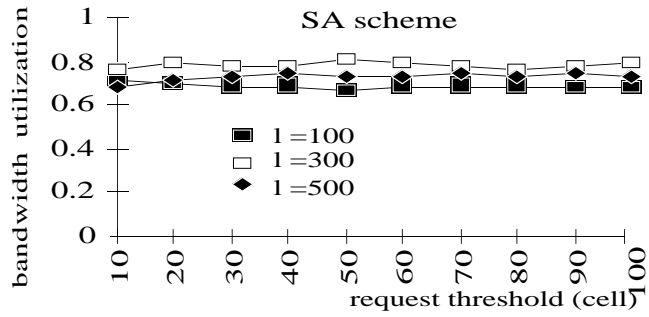

a) $\mu$ vs $1, M=1-50$ cells

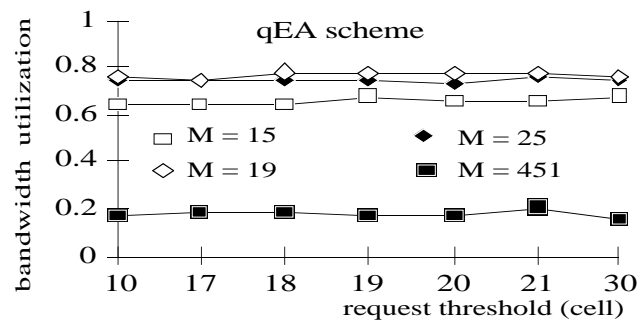

b) $\mu$ vs $M, 1=500$ cells

Figure 6 Effect of request threshold to bandwidth utilization

Figures $8 \sim 9$ show the performance (bandwidth utilization, mean response time and mean cell loss rate) for different buffer sizes (BS) and different burst lengths (BL). Generally, when the buffer is large enough, the influence of $\mathrm{BL}$ becomes weak in SA, but this is not the case in qEA. It is because $M$ is set to the maximum in SA so that a node can get a larger quota in each cycle. After finishing the quota, the node needs to wait for a long time to get another quota. During this period, the large buffer can be used to smooth the traffic. However, qEA adopts the average setting so that a node can usually get a quota in each cycle. This makes qEA sensitive to traffic burstiness. Therefore, qEA with the average setting is more suitable for less bursty traffic.
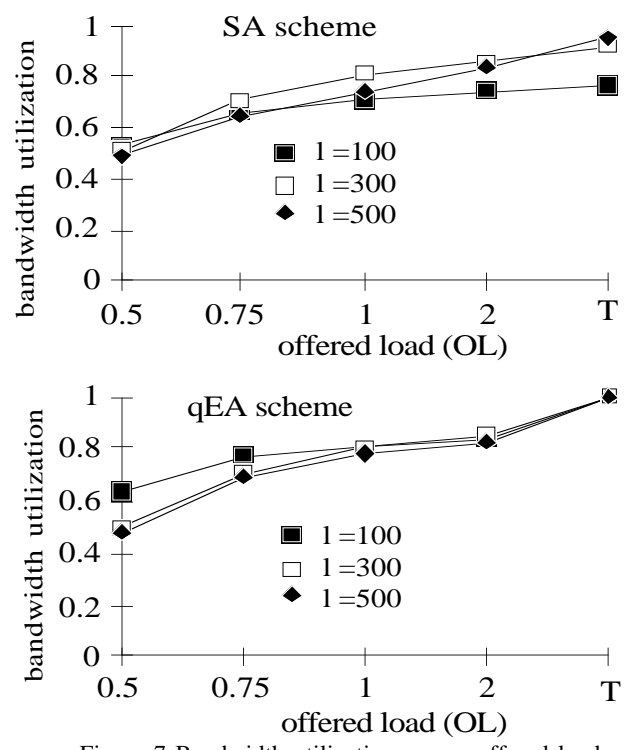

Figure 7 Bandwidth utilization versus offered load
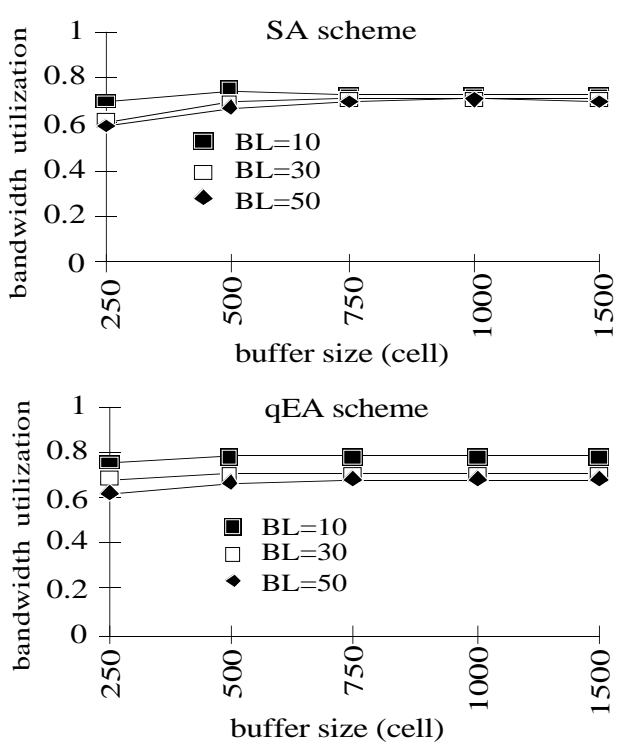

Figure 8 Bandwidth utilization versus buffer size
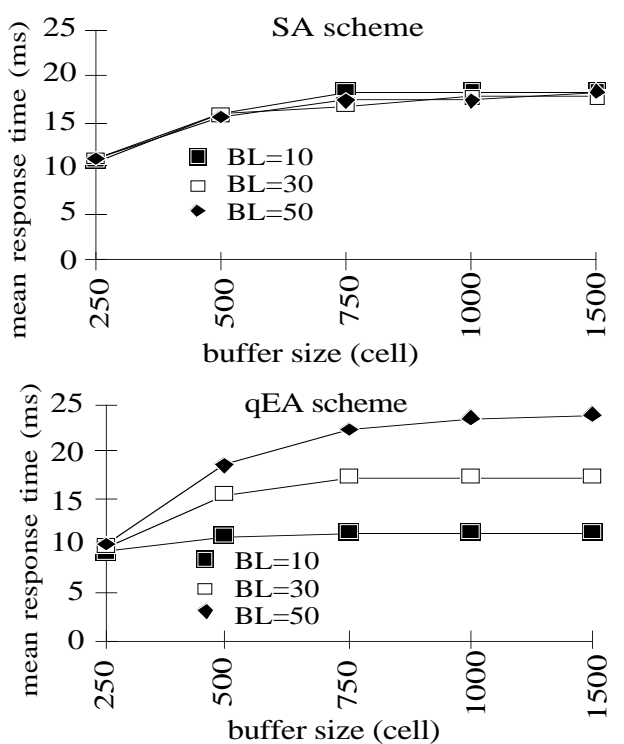

Figure 9 Mean response time versus buffer size

Figure 9 shows the mean response time (MRT) as a function of buffer size. MRT is the elapsed time between the cell arrival to the buffer and its departure. Different from SA, MTR in qEA is sensitive to BL due to the reason mentioned above. Figure 10 presents the mean cell loss rate (MCLR) calculated in terms of total lost cells over total generated cells. When the buffer size is small, MCLR for qEA is smaller than that for SA because the bandwidth allocation in the cycle in qEA with the average setting is more suitable than that in SA with the maximum setting for the traffic accumulated in a small buffer. The effect is reversed in the case of large buffers due to the reason mentioned above.

Figure 11 shows bandwidth utilization $(\mu)$ versus network size (NS) with cycle lengths of 300 and 500 cells. SA_t and EA_t are respectively the theoretical maximum $\mu$ for SA and EA respectively (refer to Section 2.2 for the calculation). It is clear that $\mu$ in SA decreases with increasing NS in the case of $l=300$. In the case of $l=500, \mu$ first increases with NS up to NS $=52$ then decreases. This is due to the mismatch between the assignment in the cycles and the traffic load from 
the nodes. Since long distance increases the response delay because of the propagation delay, this gives some time for the nodes to accumulate traffic. Similar observation is also found in qEA. However, this increase is limited by the theoretical maximum value. Anyway, qEA is less influenced by NS than SA so qEA is more suitable for metropolitan area of networks.
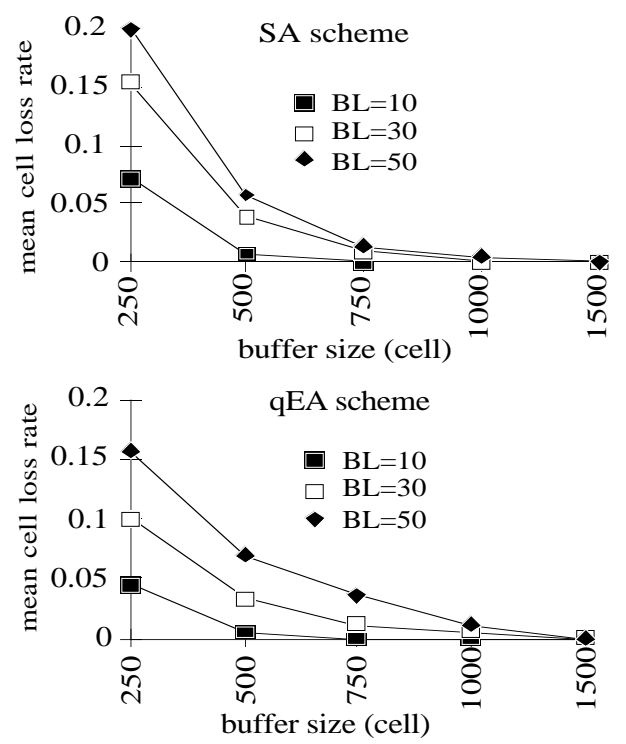

Figure 10 Mean cell loss rate versus buffer size
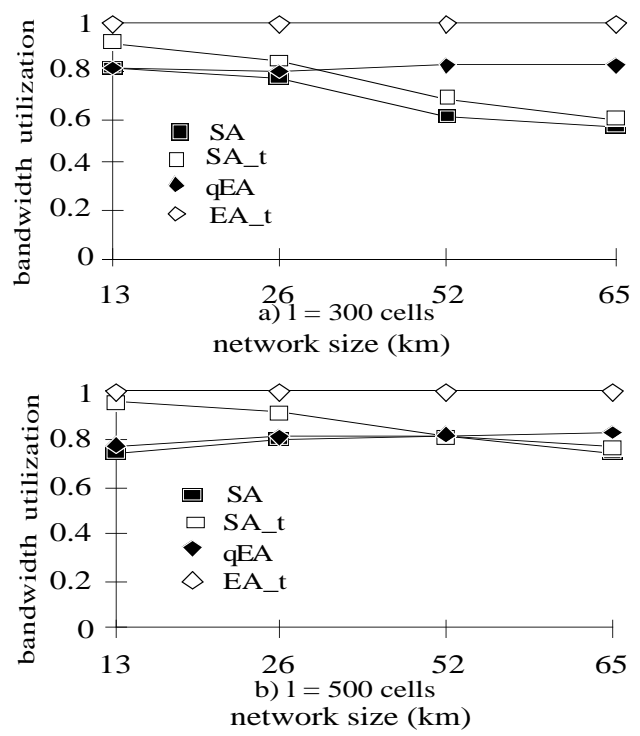

Figure 11 Bandwidth utilization versus network size

The fairness with which the nodes share the upstream bandwidth depends on the service discipline used by the scheduling algorithm at the headend. In the simulation, the nodes are served one by one in round-robin rather than FIFO discipline since the latter will lead to unfairness caused by the propagation delay between the nodes and the headend. Figure 12 plots the mean access interval for each node, which is the mean interval between two consecutive accesses to the media for a node. As shown in Figure 12.a, qEA behaves better than SA since qEA use the average setting and SA uses the maximum setting. As illustrated in Figure 12.b, SA performs very well in the case of burst length equal to 1 . A better service discipline is needed to ensure fairness among all nodes for $\mathrm{BL}>1$.

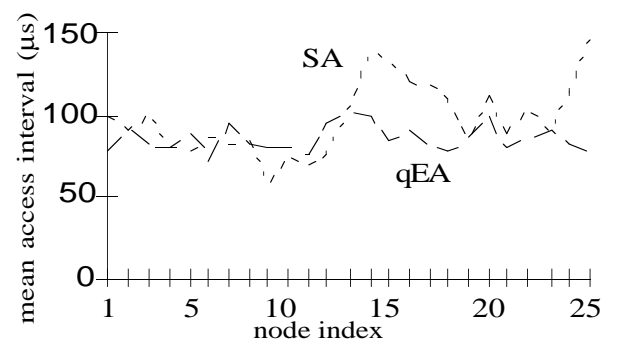

a) $1=500$ cells, $\mathrm{NS}=65 \mathrm{~km}, \mathrm{BL}=30$ cells

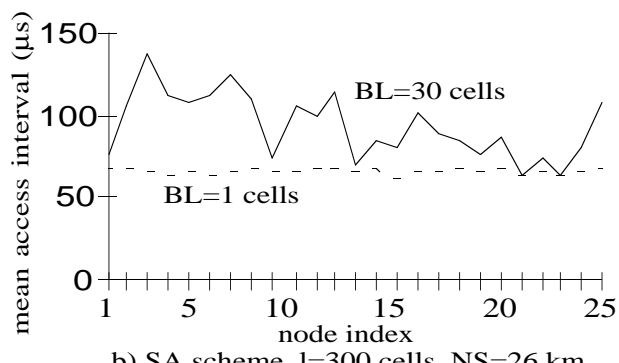

b) SA scheme, $\mathrm{l}=300$ cells, $\mathrm{NS}=26 \mathrm{~km}$

Figure 12 Fairness issue on mean access interval in SA and qEA

\section{Discussion}

A MAC scheme based on time division for the HFC system, SCCA/TD, was discussed in this paper. The reservation scheme with self-control-cyclic-access mechanism, which can be easily implemented with timers, allows SCCA/TD to provide the bounded delay and throughput. This scheme requires less control from the network and permits the nodes to be passively linked to the buses which enhance network robustness. The non-slotted media based framework can accommodate different transmission rates and support different types of MAC protocol data units. Although the priority mechanism has not been addressed, it can be easily implemented since the allocation policy is centrally performed by the headend. The static reservation segment (SRS) in each cycle can provide quick access to the medium. The scheduling algorithm is the key in SCCA/TD. Two schemes were discussed in the paper: the simple one (SA) is suitable for small size networks and the efficient one (EA) suitable for a wide range of network sizes. More efficient schemes to realize EA require further studies.

\section{References}

[1] J. W. Eng, J. F. Mollenauer, "IEEE Project 802.14: Standards for Digital Convergence", IEEE Communications Magazine, May 1995 Vol. 33 No. 5, pp2023

[2] Mario P. Vecchi, "Broadband Networks and Services: Architecture and Control", IEEE Communications Magazine, August 1995 Vol.33 NO.8, pp24-32 [3] Kazuo Imai,Tadashi Ito,Hideki Kasahara et al., "ATMR: Asynchronous transfer mode ring protocol", Computer Networks and ISDN Systems 26 (1994) 785-798 [4] J.L. Adams, "Orwell", Computer Networks and ISDN Systems 26 (1994) 771784

[5] James Martin, "Local Area Networks, Architectures and Implementations", 2nd Edition, PTR PRENTICE HALL, 1994

[6] E.A. Zurfluh, R.D. Cideciyan et al, "The IBM Zurich Research Laboratory's 1.13 Gb/s LAN / MAN Prototype", Computer Networks and ISDN Systems 26 (1993) Gb/s LAN $163-183$

[7] Bandula W.Abeysundara, "High-speed local area network and their [7] Bandula W.Abeysundara, "High-speed local area network and their
performance:a survey", ACM computing surveys,Vol.23, No.2,June 1991,p221-65 [[8] Mischa Schwartz, "Telecommunication Networks, Protocols, Modeling and Analysis", Adison-Wesley publishing Company, 1988

[9] Alta Group of Cadence Design Systmes (Inc), BONeS DESIGNER Core Library Reference, V 3.0 